\title{
Transfer of Polycyclic Aromatic Hydrocarbons to Fetuses and Breast Milk of Rats Exposed to Diesel Exhaust
}

\author{
Yoshiko Tozuka, ${ }^{a}$ Nobue Watanabe, ${ }^{b}$ Masanobu Osawa, ${ }^{b}$ Akira Toriba, ${ }^{a}$ Ryoichi Kizu, ${ }^{a}$ \\ and Kazuichi Hayakawa*,a
}

${ }^{a}$ Division of Pharmaceutical Sciences, Graduate School of Natural Science and Technology, Kanazawa University, Kakuma-machi, Kanazawa, Ishikawa 920-1192, Japan and ${ }^{b}$ Department of Environmental Health, Tokyo Metropolitan Public Health Research Institute, 3-24-1, Hyakunin-chyo, Shinjyuku-ku, Tokyo 169-0073, Japan

(Received May 22, 2004; Accepted July 9, 2004; Published online July 23, 2004)

\begin{abstract}
Polycyclic aromatic hydrocarbons (PAHs) were analyzed in maternal blood and fetuses from Fischer 344 rats exposed to diesel exhaust (DE) during pregnancy, and in breast milk from rats exposed to DE during pregnancy and lactation using high performance liquid chromatography with fluorescence detection. Concentrations of phenanthrene (Phe), anthracene (Ant) and benz $[a]$ anthracene $(\mathrm{BaA})$ were significantly higher in maternal blood of the DE group than those of the control group. Concentration of Phe in fetuses of the DE group was significantly higher than those of the control group. Concentrations of fluorene, Ant, fluoranthene (Flu), pyrene (Pyr), BaA and chrysene (Chr) tended to be higher in fetuses of the DE group. The levels of Ant, Flu, Pyr and Chr in breast milk from the DE group were significantly higher than those of the control group. These results indicate that PAHs taken into mother rat by the inhalation of DE are transferred into fetuses via placenta and into breast milk. This is the first report to clarify the transportation of inhaled PAHs into fetuses and breast milk from mother rats.
\end{abstract}

Key words — polycyclic aromatic hydrocarbon, diesel exhaust, endocrtine disruption, transplacenta, fetus, breast milk

\section{INTRODUCTION}

Inhalation of diesel exhaust (DE) has been shown to interfere with reproduction in mice and rats, as reflected by decreased daily sperm production, degeneration of Leydig cells, and abnormal delivery. ${ }^{1-4)}$ Indirect exposure of mothers to DE has been reported to affect the differentiation and formative periods of the fetal testis, ovary and thymus. ${ }^{5)}$

Particulates in DE adsorb many chemicals, such as polycyclic aromatic hydrocarbons (PAHs). ${ }^{6-9)} \mathrm{We}$ previously showed that several PAHs and monohydroxy PAHs (OHPAHs) have estrogenic/antiestrogenic and antiandrogenic activities in vitro. ${ }^{10-13)}$ Though the inhalation of DE during the formative period affects the differentiation process of the fetal organs, little is known about whether PAHs transfer

\footnotetext{
*To whom correspondence should be addressed: Division of Pharmaceutical Sciences, Graduate School of Natural Science and Technology, Kanazawa University, Kakuma-machi, Kanazawa 920-1192, Japan. Tel. \& Fax: +81-76-234-4413; Email: hayakawa@p.kanazawa-u.ac.jp
}

from mother to offspring and act as endocrine disrupters in the offspring.

The present study examined the effects of indirect exposure of mothers to DE. PAHs were measured in maternal blood, fetuses and breast milk to determine whether PAHs that are present in DE are transferred from mothers to fetuses and neonates.

\section{MATERIALS AND METHODS}

Generation of DE — DE was generated by a running diesel engine (309 cc, Model NFAD-50, Yanmar Co., Osaka, Japan). DE was diluted with clean air in a dilution tunnel and then drawn into an inhalation chamber. The exchange rate of the chamber was 15 times/hr. Concentrations of particulate matter $\left(1.73 \mathrm{mg} / \mathrm{m}^{3}\right)$ and nitrogen dioxide $(0.80 \mathrm{ppm})$ were monitored by an automatic $\beta$-ray dust-mass monitor (Model BAM-102, Shibata Scientific Technology Co., Tokyo, Japan) and a chemiluminescent analyzer (Model 8440, Monitor Labs Co., San Diego, CA, U.S.A.), respectively. 


\section{Animal Treatments}

Thirteen pregnant

Fischer 344 rats at the 6th day of gestation were obtained from Charles River Japan (Kanagawa, Japan). After the rats were housed for a day, they were divided into DE $(n=7)$ and control $(n=6)$ groups. The exposure period of DE was $6 \mathrm{hr}$ daily from 10 a.m. to 4 p.m., for 2 weeks from the 7th day (Tue.) to the 20th day (Mon.) of gestation except for four days (Sat. and Sun.). Rats of each group were maintained in an inhalation chamber $\left(1.6 \mathrm{~m}^{3}\right) \mathrm{kept}$ at 24 $\pm 2{ }^{\circ} \mathrm{C}$ and $55 \pm 5 \%$ humidity. The daily illumination time program was $12 \mathrm{hr}$ light and $12 \mathrm{hr}$ dark. All rats were allowed free access to food (NMF, Oriental Yeast Co. Ltd, Tokyo, Japan) and water. Both fetuses and maternal blood were taken from each rat after finishing all DE exposure on the 20th day of gestation. These samples were stored at $-80^{\circ} \mathrm{C}$ until analysis. The average body weights of the mothers of the DE and control groups were $218.6 \pm 10.2 \mathrm{~g}$ and $213.0 \pm 14.3 \mathrm{~g}$, respectively. The number of fetuses per mother ranged from 7 to 11 , and the mean numbers per mother in the DE and control groups were 8.71 and 8.67 , respectively. The average body weights of male and female fetuses of the DE and control groups were $3.40 \pm 0.24 \mathrm{~g}, 3.24 \pm 0.18 \mathrm{~g}$, $3.42 \pm 0.23 \mathrm{~g}$ and $3.13 \pm 0.20 \mathrm{~g}$, respectively.

To determine the transfer of PAHs to neonates via breast milk, pregnant Fischer 344 rats were housed for a day and divided into DE $(n=7)$ and control $(n=6)$ groups. The DE exposure period was from the 7th day of gestation until the 14th day after birth. The exposure conditions were the same as in the above experiment. At the 14th day after birth, breast milk was collected from each rat after subcutaneous injection of prolactin under anesthesia using diethylether. The milk samples were stored at $-80^{\circ} \mathrm{C}$ until analysis.

The treatment and care of the rats were carried out according to the protocol approved by Animal Care and Use Committee of the Tokyo Metropolitan Public Health Research Institute in a facility approved by the Japan Association for Accreditation of Laboratory Animal Care.

Preparation of Biological Samples — After weighing fetuses (11.9-13.3 g) or measuring the volume of maternal blood (5.6-6.2 $\mathrm{ml})$, the samples were refluxed with $5 \mathrm{M}$ potassium hydroxide/ethanol $(1: 4, \mathrm{v} / \mathrm{v})$ solution $(5 \mathrm{ml} / \mathrm{g}$ fetuses or $3 \mathrm{ml} / \mathrm{ml}$ blood) in a water bath at about $95^{\circ} \mathrm{C}$ for $2 \mathrm{hr}$. As described previously, ${ }^{14)}$ naphthalene- $d_{8}$, acenaphthene- $d_{10}$, phenanthrene- $d_{10}$, pyrene- $d_{10}$ and benzo $[a]$ pyrene- $d_{12}$ (Wako Pure Chemicals, Tokyo,
Japan) were spiked to the above solution as internal standards. Both PAHs and internal standards in the mixture were extracted three times with an equivalent volume of $n$-hexane. The $n$-hexane solutions were combined and washed three times with distilled water. Then the solution was dried with anhydrous sodium sulfate and was applied to a Sep-Pak Plus Silica cartridge (Waters, Milford, MA, U.S.A.). Both PAHs and internal standards were eluted with $20 \mathrm{ml}$ of $n$-hexane. After the addition of $20 \mu \mathrm{l}$ of DMSO, the eluate was completely evaporated under a stream of nitrogen. The residue was redissolved in $180 \mu \mathrm{l}$ of acetonitrile for HPLC analysis.

Breast milk samples were pre-treated according to the association of official analytical chemists (AOAC) method for dioxins in foods ${ }^{15)}$ with several modifications. Each breast milk sample (1.0-1.8 ml) was transferred to a vessel and added with naphthalene- $d_{8}$, acenaphthene- $d_{10}$, phenanthrene- $d_{10}$, pyrene$d_{10}$ and benzo[ $\left.a\right]$ pyrene- $d_{12}$ as internal standards. ${ }^{14)}$ The solution was mixed with a $54 \mathrm{mM}$ potassium oxalate, and then added with an equivalent volume of ethanol. Lipid was extracted from breast milk three times with two volumes of $n$-hexane/ diethylether $(1: 1, \mathrm{v} / \mathrm{v})$. The extraction solvent was removed completely under a stream of nitrogen. The amount of lipid was determined gravimetrically and refluxed with $5 \mathrm{ml}$ of $5 \mathrm{M}$ potassium hydroxide/ethanol $(1: 4, \mathrm{v} / \mathrm{v})$ for $2 \mathrm{hr}$ in a water bath at about $95^{\circ} \mathrm{C}$. Both PAHs and internal standards were extracted three times with an equivalent volume of $n$-hexane. The $n$-hexane solutions were combined and washed three times with distilled water and dried with anhydrous sodium sulfate. The solution was applied to a Sep-Pak Plus Silica cartridge. Both PAHs and internal standards were eluted with $20 \mathrm{ml}$ of $n$-hexane. After the addition of $20 \mu \mathrm{l}$ of DMSO, the eluate was completely evaporated under a stream of nitrogen. The residue was redissolved in $180 \mu \mathrm{l}$ of acetonitrile for HPLC analysis.

HPLC Analysis — PAHs were determined by HPLC with fluorescence detection according to our previous method. ${ }^{14)}$ The HPLC system consisted of LC-10AD intelligent pumps, an SIL-10A automatic injector, a RF-10AXL fluorescence detector and an SCL-10A system controller (all from Shimadzu, Kyoto, Japan). PAHs were separated using a guard column (Inertsil ODS-P, $10 \times 4.0 \mathrm{~mm}$ i.d., $5 \mu \mathrm{m}$; GL Sciences, Tokyo, Japan) and an analytical column (Inertsil ODS-P, $250 \times 4.6 \mathrm{~mm}$ i.d., $5 \mu \mathrm{m}$; GL Sciences). The mobile phase was a mixture of acetonitrile/water. The acetonitrile concentration was in- 
creased by a combination of stepwise and gradient elutions as follows: $55 \%$ (0-20 $\mathrm{min}$ ), $70-80 \%$ (20$35 \mathrm{~min}$, linear gradient), $90 \%$ (35-45 $\mathrm{min}$ ), 90-100\% (45-60 min, linear gradient), 100\% (60-80 min). Fluorescence detection wavelengths $(\mathrm{Ex} / \mathrm{Em})$ were 280/340 nm (0-30 min) for naphthalene (Nap), acenaphthene (Ace), fluorene (Fle), phenanthrene$d_{10}$ and phenanthrene (Phe), 250/400 nm (30$33.5 \mathrm{~min}$ ) for anthracene (Ant), 286/433 nm (33.5$36 \mathrm{~min}$ ) for fluoranthene (Flu), 331/392 nm (36$40 \mathrm{~min}$ ) for pyrene- $d_{10}$ and pyrene (Pyr), 264/407 nm (40-66.5 min) for benz[a]anthracene $(\mathrm{BaA})$, chrysene $(\mathrm{Chr})$, benzo[b]fluoranthene $(\mathrm{BbF})$, benzo $[k]$ fluoranthene $(\mathrm{BkF})$, benzo $[a]$ pyrene- $d_{12}$, benzo $[a]$ pyrene $(\mathrm{BaP}), \operatorname{dibenz}[a, h]$ anthracene (DBA) and benzo[ghi]perylene (BghiP), and 294/ $482 \mathrm{~nm}(66.5-80 \mathrm{~min})$ for indeno[1,2,3-cd]pyrene (IDP). The peak height method was used for the quantification by using naphthalene- $d_{8}$ for Nap, phenanthrene- $d_{10}$ for Ace, Fle, Phe and Ant, pyrene$d_{10}$ for Flu, Pyr, BaA and Chr and benzo[ $\left.a\right]$ pyrene$d_{12}$ for BbF, BkF, BaP, DBA, BghiP and IDP as internal standards.

\section{Analysis of PAHs in the Air of Inhalation Cham-} ber - Suspended diesel exhaust particulates (DEP) in the inhalation chamber were collected on two filters (Empore Extraction Disk $\mathrm{C}_{18}$ Fast Flow $\phi$ $47 \mathrm{~mm}, 3 \mathrm{M}$, St. Paul, MN, U.S.A.) put in the filter holder, which were previously spiked with $1 \mathrm{mg}$ of ascorbic acid to prevent oxidation of PAHs, at a flow rate of $1.0 \mathrm{l} / \mathrm{min}$ for $6 \mathrm{hr}$ during the exposure to DE (about $450 \mathrm{l} / \mathrm{sample}$ ). PAHs on collected DEP were determined by GC (HP 5890 Series II, Hewlett Packard, Palo Alto, CA, U.S.A.)-MS (HP 5971A MSD) in the electron impact (EI) ionization mode using selected ion monitoring (SIM) mode as follows. The filters were extracted with $5 \mathrm{ml}$ of dichloromethane by sonication for $10 \mathrm{~min}$. The supernatant was separated from the mixture by centrifugation (2500 rpm, $10 \mathrm{~min})$. In the subsequent preparation, the solution was divided into two sample solutions, because the concentration difference between PAHs having two or three rings and those having more rings was very large. The one sample solution was added with chrysene- $d_{12}(0.1 \mathrm{ppm}$ as final concentration) as an internal standard for GCMS analysis of PAHs having two and three rings. In order to analyze PAHs having four rings and more, $2 \mathrm{ml}$ of the other sample solution was dried under a stream of nitrogen, and then dissolved in $0.2 \mathrm{ml}$ of $n$-hexane. The solution was applied to a Sep-Pak Plus Silica cartridge (Waters, Milford, MA, U.S.A.)
Table 1. Concentrations of PAHs in the Inhalation Chamber Air

\begin{tabular}{cc}
\hline \hline Compound & Concentration $\left(\mathrm{ng} / \mathrm{m}^{3}\right)$ \\
\cline { 2 - 2 } & Mean \pm S.D. \\
\hline Ace & $150 \pm 34$ \\
Fle & $3160 \pm 401$ \\
Phe & $2280 \pm 291$ \\
Ant & $70.3 \pm 10.9$ \\
Flu & $148 \pm 19$ \\
Pyr & $133 \pm 5$ \\
BaA & $17.2 \pm 2.7$ \\
Chr & $39.3 \pm 6.8$ \\
BbF & $9.9 \pm 2.1$ \\
BkF & $4.9 \pm 1.1$ \\
BaP & $3.7 \pm 0.5$ \\
DBA & $<1.4$ \\
BghiP & $<6.0$ \\
IDP & $4.2 \pm 0.1$
\end{tabular}

Ace; acenaphthene, Fle; fluorene, Phe; phenanthrene, Ant; anthracene, Flu; fluoranthene, Pyr; pyrene, BaA; benz $[a]$ anthracene, Chr; chrysene, BbF; benzo[b]fluoranthene, $\mathrm{BkF}$; benzo[k]fluoranthene, BaP; benzo $[a]$ pyrene, DBA; dibenz $[a, h]$ anthracene, BghiP; benzo[ghi]perylene, IDP; indeno[1,2,3-cd]pyrene.

washed with $5 \mathrm{ml}$ of $n$-hexane and dichloromethane, respectively, before use. PAHs having four rings and more were eluted with $5 \mathrm{ml}$ of dichloromethane after eluting with $4 \mathrm{ml}$ of $n$-hexane to wash PAHs having two and three rings. The solvents in the dichloromethane eluate were removed completely under a steam of nitrogen and dissolved in $0.2 \mathrm{ml}$ of toluene containing chrysene- $d_{12}(0.1 \mathrm{ppm})$ as an internal standard for GC-MS analysis.

\section{RESULTS}

The concentrations of PAHs in DE air in the chamber are listed in Table 1. Among the fifteen PAHs, seven PAHs (Fle, Phe, Ant, Flu, Pyr, BaA and Chr) were quantified in maternal blood. The levels of Phe, Ant and BaA in the DE group were significantly higher than those in the control group (Fig. 1). Fle, Flu, Pyr and Chr were higher in the DE group than those in the control group, although the differences were not significant. Nap, two-ring PAH, was not quantified because of low recovery in sample preparation. PAHs having five or six rings were not detected.

$\mathrm{Fle}$, Phe, Ant, Flu, Pyr, BaA and Chr were quantified in fetuses. The level of Phe in the DE group was significantly higher than that in the control group 


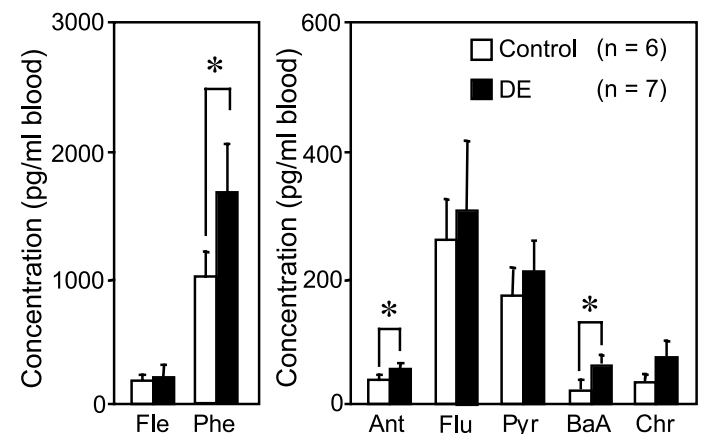

Fig. 1. Concentrations of PAHs in Maternal Blood

Error bars indicate S.D. Asterisks indicate significant differences between $\mathrm{DE}$ and control groups $(p<0.05)$.

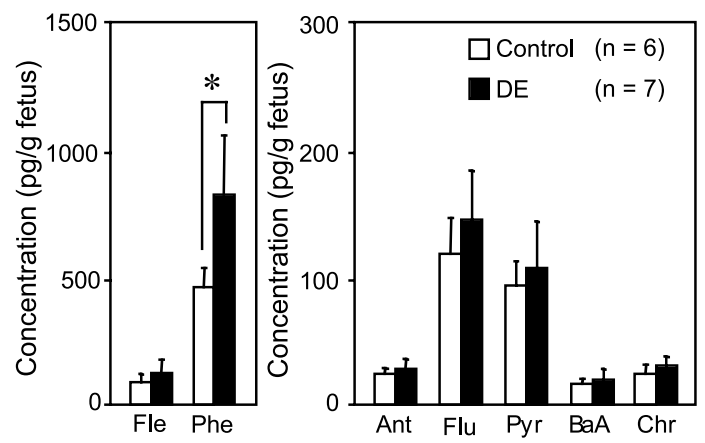

Fig. 2. Concentrations of PAHs in Fetus

Error bars indicate S.D. Asterisk indicates a significant difference between $\mathrm{DE}$ and control groups $(p<0.05)$.

(Fig. 2). Concentrations of Fle, Ant, Flu, Pyr, BaA and $\mathrm{Chr}$ in the DE group tended to be higher than those in the control group, although they were not significant. Nap, two-ring PAH, was not quantified because of low recovery in sample preparation. PAHs having five or six rings were not detected.

Ace, Fle, Phe, Ant, Flu, Pyr, BaA and Chr were quantified in breast milk. The levels of Ant, Flu, Pyr and $\mathrm{Chr}$ showed significantly higher in the DE group than those in the control group (Fig. 3). The concentration of $\mathrm{BaA}$ of the $\mathrm{DE}$ group tended to be approximately 4 times as high as that of control group, although the difference was not significant. Ace, Fle and Phe concentrations were not different between the DE and control groups. The other PAHs were not quantified or detected due to low recovery as described above.

PAHs in maternal livers of DE and control groups were not significantly different (data not shown). When rats were housed in clean air after exposure to DE during pregnancy, no increase in PAH concentrations was observed (data not shown).

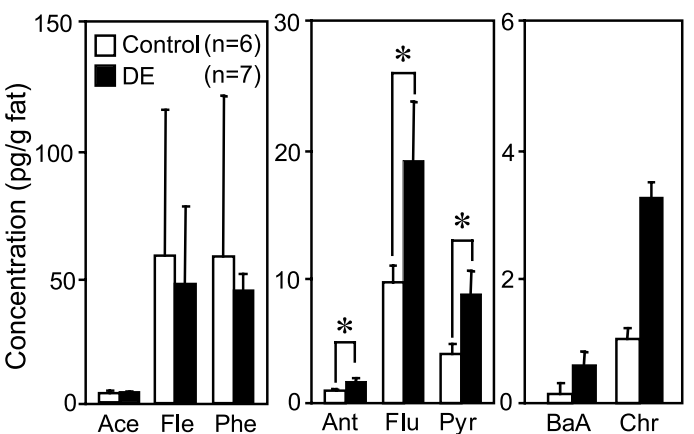

Fig. 3. Concentrations of PAHs in Breast Milk

Error bars indicate S.D. Asterisks indicate significant differences between DE and control groups $(p<0.05)$.

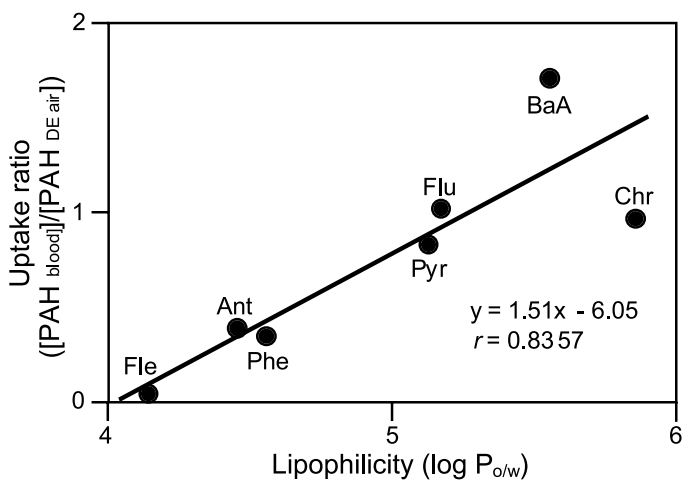

Fig. 4. Effect of Lipophilicity (Expressed as $\log \mathrm{P}_{\mathrm{o} / \mathrm{w}}$ ) on Uptake of PAHs by Maternal Blood (Expressed as Ratio of Concentrations in Maternal Blood and DE)

\section{DISCUSSION}

The levels of Phe, Ant and BaA in maternal blood (Fig. 1), Phe in fetuses (Fig. 2) and Ant, Flu, Pyr and Chr in breast milk (Fig. 3) of the DE group were significantly higher than those of the control group. Although, maternal blood, fetuses and breast milk in the control group also contained PAHs to some extents which might mainly come from foods, these results showed that inhaled PAHs were transferred from mother to the next generation. This is the first report to show that PAHs are transferred to fetuses via the placenta and to new born rats from breast milk of mothers exposed to diesel exhaust.

Figure 4 shows the relationship between the ratio of the concentration of a PAH having three or four rings in maternal blood to its concentration in $\mathrm{DE}$ air and the distribution coefficient of the PAH in $n$-octanol and water $\left(\log \mathrm{P}_{\mathrm{o} / \mathrm{w}}\right){ }^{16)}$ Lipophilicity of a PAH was expressed as $\log \mathrm{P}_{\mathrm{o} / \mathrm{w}}$ which is the distribution coefficient of the PAH in $n$-octanol and water. 


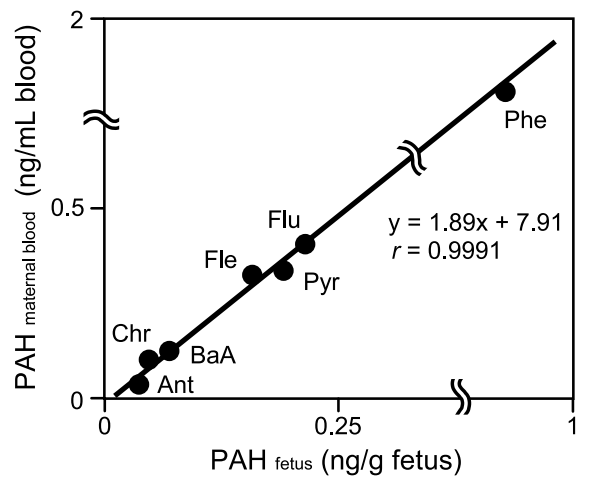

Fig. 5. Relationship between Concentrations of PAHs in Fetuses and Maternal Blood

The uptake ratio was expressed as the ratio of the concentration of a PAH in maternal blood to its concentration in DE air. A plot of these data shows that lipophilic PAHs have a higher affinity for blood. The concentrations of PAHs in fetuses was closely correlated with those in maternal blood (Fig. 5). These results suggest that fetal exposure to PAHs strongly depends on the concentrations in the blood of the mother.

As indicated in Fig. 3, PAHs were transferred to breast milk. The transfer of PAHs from mother to suckling via breast milk suggests the strong possibility of endocrine disrupting effect of DE on the next generation even after birth. Breast milk is one of the excretion routes of lipophilic compounds from a body because milk is rich of lipid.

The concentration levels of these PAHs in DE were more than an order of magnitude lower than those of PAHs having 4 or lesser rings (Table 1). This might be the main reason for the inability to quantitate PAHs in maternal blood, fetuses and breast milk. However, the concentrations of PAHs in DE used in this work were in the ranges of their atmospheric concentration in downtown Kanazawa, Japan. ${ }^{17)}$ The other PAHs were not determined or detected because of low recovery in sample preparation.

Our findings that PAHs in maternal livers of DE and control groups were not significantly different and that exposure to clean air after exposure to DE during pregnancy didn't increase PAH concentrations may be due to rapid metabolism and elimination of PAHs. ${ }^{18-20)}$ PAHs are metabolized to phenols (OHPAHs), diols and epoxides and large parts of both phenols and diols are thoroughly conjugated with glucuronic acid and sulfuric acid to be excreted in urine or bile. Especially, several OHPAHs exhib- ited agonistic or antagonistic effects in the binding process to estrogen receptor. ${ }^{21)}$ These facts strongly suggest that not only PAHs but also their metabolites could affect the differentiation processes of growing rats. Further studies are needed to investigate the endocrine disrupting effects of PAH metabolites after the inhalation of DE.

In conclusion, PAHs taken into the maternal body by the inhalation of DE were transferred to the fetus via the placenta; PAHs taken into the maternal body by the inhalation of DE were transferred to breast milk.

Acknowledgements This research was a part of the international project of the 21st Century COE Program "Long- and Short-Term Dynamics of PanJapan Sea Area: Environmental Monitoring and Prediction." This research was also supported by a Grant-in-Aid for Scientific Research from the Ministry of Education, Science, Sports and Culture, Japan.

\section{REFERENCES}

1) Yoshida, S., Sagai, M., Oshino, S., Umeda, T., Ihara, T., Sugamata, M., Sugawara, I. and Takeda, K. (1999) Exposure to diesel exhaust affects the male reproductive system of mice. Int. J. Androl., 22, 307315.

2) Watanabe, N. and Oonuki, Y. (1999) Inhalation of diesel engine exhaust affects spermatogenesis in growing male rats. Environ. Health Perspect., 107, 539-544.

3) Tsukue, N., Toda, N., Tsubone, H., Sagai, M., Jin, W. Z., Watanabe, G., Taya, K., Birumachi, J. and Suzuki, A. K. (2001) Diesel exhaust (DE) affects the regulation of testicular function in male Fischer 344 rats. J. Toxicol. Environ. Health A, 63, 115-126.

4) Tsukue, N., Tsubone, H. and Suzuki, A. K. (2002) Diesel exhaust affects the abnormal delivery in pregnant mice and the growth of their young. Inhal. Toxicol., 14, 635-651.

5) Watanabe, N. and Kurita, M. (2001) The masculinization of the fetuses during pregnancy due to inhalation of diesel exhaust. Environ. Health Perspect., 109, 111-119.

6) Yergey, J. A., Risby, T. H. and Lestz, S. S. (1982) Chemical characterization of organic adsorbates on diesel particulate matter. Anal. Chem., 54, 354-357.

7) Schuetzle, D., Rlley, T. L., Prater, T. J., Harvey, T. M. and Hunt, D. F. (1982) Analysis of nitrated polycyclic aromatic hydrocarbons in diesel particulates. 
Anal. Chem., 54, 265-271.

8) Schuetzle, D. (1983) Sampling of vehicle emission for chemical analysis and biological testing. Environ. Health Perspect., 47, 65-80.

9) Hampton, C. V., Pierson, W. R., Schuetzle, D. and Harvey, T. M. (1983) Hydrocarbon gases emitted from vehicles on the road. 2. Determination of emission rates from diesel and spark-ignition vehicles. Environ. Sci. Technol., 17, 699-708.

10) Kizu, R., Okamura, K., Toriba, A., Mizokami, A., Burnstein, K. L., Klinge, C. M. and Hayakawa, K. (2003) Antiandrogenic activities of diesel exhaust particle extracts in PC3/AR human prostate carcinoma cells. Toxicol. Sci., 76, 299-309.

11) Kizu, R., Okamura, K., Toriba, A., Kakishima, H., Mizokami, A., Burnstein, K. L. and Hayakawa, K. (2003) A role of aryl hydrocarbon receptor in the antiandrogenic effects of polycyclic aromatic hydrocarbons in LNCaP human prostate carcinoma cells. Arch. Toxicol., 77, 335-43.

12) Hirose, T., Morito, K., Kizu, R., Toriba, A., Hayakawa, K., Ogawa, S., Inoue, S., Muramatsu, M. and Masamune, Y. (2001) Estrogenic/antiestrogenic activities of benzo $[a]$ pyrene monohydroxy derivatives. J. Health Sci., 47, 552-558.

13) Hayakawa, K., Onoda, Y., Kamiya, M., Hirose, T., Toriba, A. and Kizu, R. (eds.) (2002) Structure-activity relationship for estrogenic/antiestrogenic activities of monohydroxylated polycyclic aromatic hydrocarbons. In The 5th Annual Meeting of Japan Society of Endocrine Disrupters Research, Hiroshima, 25-26, Nov., 2002, Japan Society of Endocrine Disrupters Research, Ibaraki, Japan, p. 123.

14) Toriba, A., Kuramae, Y., Chetiyanukornkul, T., Kizu, R., Makino, T., Nakazawa, H. and Hayakawa, K. (2003) Quantification of polycyclic aromatic hydro- carbons (PAHs) in human hair by HPLC with fluorescence detection: a biological monitoring method to evaluate the exposure to PAHs. Biomed. Chromatogr., 17, 126-132.

15) Williams, S. (Ed.) (1984) method 16.064 and 20.012. In Official methods of analysis of the association of official analytical chemists, 14th Edition (Williams, S., ed.), AOAC, Virginia, U.S.A.

16) WHO (1998) Selected non-heterocyclic polycyclic aromatic hydrocarbons. In Environmental Health Criteria 202 (Inter-Organization Programme for the Sound Management of Chemicals, ed.), World Health Organization, Geneva, Switzerland, pp. 2226.

17) Hayakawa, K., Murahashi, T., Akutsu, K., Kanda, T., Tang, N., Kakimoto, H., Toriba, A. and Kizu, R. (2000) Comparison of polycyclic aromatic hydrocarbons and nitropolycyclic aromatic hydrocarbons in airborne and automobile exhaust particulates. Polycycl. Aromat. Comp., 20, 179-190.

18) Withey, J. R., Burnett, R., Law, F. C., Abedini, S. and Endrenyi, L. (1994) Pharmacokinetics of inhaled pyrene in rats. J. Toxicol. Environ. Health, 43, 103116.

19) Mitchell, C. E. (1983) The metabolic fate of benzo $[a]$ pyrene in rats after inhalation. Toxicology, 28, 65-73.

20) Kadry, A. M., Skowronski, G. A., Turkall, R. M. and Abdel-Rahman, M. S. (1995) Comparison between oral and dermal bioavailability of soiladsorbed phenanthrene in female rats. Toxicol. Lett., 78, 153-63.

21) Nishihara, T., Nishikawa, J., Kanayama, T., Dakeyama, F., Saito, K., Imagawa, M., Takatori, S., Kitagawa, Y., Hori, S. and Utsumi, H. (2000) Estrogenic activities of 517 chemicals by yeast two-hybrid assay. J. Health Sci., 46, 282-298. 